\title{
BREAST IMAGING REPORTING AND DATA SYSTEM (BI-RADS $\left.{ }^{\text {TM }}\right)$ : COMO TEM SIDO UTILIZADO?*
}

\author{
Eduardo Rodrigues Godinho' ${ }^{1}$ Hilton Augusto $\mathrm{Koch}^{2}$
}

\begin{abstract}
Resumo O Breast Imaging Reporting and Data System (BI-RADS ${ }^{\mathrm{TM}}$ ), do American College of Radiology, foi concebido para padronizar o laudo mamográfico e reduzir os fatores de confusão na descrição e interpretação das imagens, além de facilitar o monitoramento do resultado final. OBJETIVO: Identificar a maneira como vem sendo utilizado o $\mathrm{BI-RADS}^{\mathrm{TM}}$, gerando informações que possam auxiliar o Colégio Brasileiro de Radiologia a desenvolver estratégias para aperfeiçoar o seu uso. MATERIAIS E MÉTODOS: Os dados foram coletados na cidade de Goiânia, GO. Foram solicitados os exames de mamografia anteriores a todas as mulheres que se dirigiram ao serviço para realização de mamografia entre janeiro/2003 e junho/2003. Foram incluídos na análise exames anteriores, realizados entre 1/7/2001 e 30/6/2003. RESULTADOS: Foram coletados 104 laudos anteriores, emitidos por 40 radiologistas de 33 diferentes serviços. Dos 104 laudos, $77 \%(n=80)$ utilizavam o BI-RADS ${ }^{\mathrm{TM}}$. Destes, apenas $15 \%(n=12)$ eram concisos, nenhum utilizava a estrutura e organização recomendadas pelo sistema, $98,75 \%$ ( $n=79)$ não respeitavam o léxico e $65 \%$ ( $n=51)$ não faziam recomendação de conduta. CONCLUSÃO: O BI-RADS ${ }^{\mathrm{TM}}$, apesar de bastante utilizado, não foi reconhecido como sistema para padronização dos laudos. Foi usado quase exclusivamente como forma de classificação final dos exames.

Unitermos: Mamografia; Neoplasias mamárias; Detecção.
\end{abstract}

Abstract Breast Imaging Reporting and Data System (BI-RADS $\left.{ }^{\mathrm{T}}\right)$ : how is it being used?

The Breast Imaging Reporting and Data System (BI-RADS ${ }^{T}$ ) from the American College of Radiology is a system designed to standardize mammographic reporting, reduce confounding factors in breast imaging interpretation, and to simplify the management of the final result. OBJECTIVE: To identify how the system is being used, providing information to help the Brazilian College of Radiology to develop strategies to improve its use. MATERIALS AND METHODS: Data were collected in the City of Goiânia, GO, Brazil. Each woman submitted to the examination in the service between January and June, 2003 was asked to provide previous reports and films. Examinations performed between 1/7/2001 and 30/6/2003 were included in the analysis. RESULTS: A total of 104 previous reports done by $\mathbf{4 0}$ radiologists from $\mathbf{3 3}$ different services were collected for analysis; $77 \%(n=80)$ used the BI-RADS ${ }^{\text {TM }}$. From these, only $15 \%(n=12)$ were concise, none followed the recommendations suggested by the system, $98.75 \%(n=79)$ were not in conformity with the lexicon terminology and in $65 \%(n=51)$ there was no reference to pertinent recommendations. CONCLUSION: Although largely used, BI-RADS ${ }^{T w}$ was not recognized as a system designed to standardize the reports and it was used almost exclusively to a final categorization of the exams.

Key words: Mammography; Breast neoplasms; Detection.

\section{FUNDAMENTOS}

O Breast Imaging Reporting and Data System (BI-RADS $\left.{ }^{\mathrm{TM}}\right)$, do American College of Radiology (ACR), foi desenvolvido para padronizar o laudo mamográfico e

* Trabalho realizado no Núcleo Radiológico e no Departamento de Radiologia da Universidade Federal do Rio de Janeiro (UFRJ) Rio de Janeiro, RJ.

1. Mestre e Doutorando em Radiologia pela Faculdade de Medicina da UFRJ.

2. Professor Titular de Radiologia da UFRJ, Coordenador da linha de pesquisa "Bases para um Programa de Detecção Precoce do Câncer de Mama por Meio da Mamografia", do Curso de Pós-Graduação em Radiologia da UFRJ.

Endereço para correspondência: Dr. Eduardo Rodrigues Godinho. Rua 5, no 784, Centro. Goiânia, GO, 74055-290. E-mail: eduardorgodinho@ig.com.br

Recebido para publicação em 22/10/2003. Aceito, após revisão, em 24/11/2003. reduzir os fatores de confusão na descrição e interpretação das imagens, além de facilitar o monitoramento do resultado final do exame $^{(1)}$. É produto de um esforço colaborativo entre membros de vários comitês do ACR, com a cooperação do National Cancer Institute, Centers for Disease Control and Prevention, Food and Drug Administration, American Medical Association, American College of Surgeons e College of American Pathologists ${ }^{(\mathbf{1}}$.

O sistema busca melhorar a qualidade do atendimento das pacientes através da auditoria e monitoramento dos resultados. Na introdução, os elaboradores do sistema chamam a atenção de que tanto os médicos assistentes como os radiologistas devem estar cientes dos benefícios e das limitações dos métodos de estudo por imagem ${ }^{(1)}$.

O BI-RADS ${ }^{\text {TM }}$ é estruturado em quatro seções: Seção I - Léxico da imagem mamográfica; Seção II - Sistematização do laudo mamográfico; Seção III - Acompanhamento e monitoramento do resultado final; Seção IV - Criação de um banco de dados nacional $^{(1)}$.

Seção I - Léxico da imagem mamográfica

Nesta seção, a Força Tarefa em Câncer de Mama padroniza os termos descritivos a serem utilizados no laudo mamográfico. Assim, os achados devem ser descritos de acordo com o léxico, que constitui uma 
espécie de regulamentação dos termos a serem utilizados para cada tipo de lesão.

No léxico são detalhados: A - Massas; B - Calcificações; C - Distorção da arquitetura; D - Casos especiais ( 1 - densidade tubular/ducto solitário dilatado; 2 - linfonodo intramamário; 3 - tecido mamário assimétrico; 4 - densidade focal assimétrica; 5 - densidade em uma incidência); E Achados associados (retração da pele, espessamento cutâneo, espessamento trabecular, lesão de pele, adenopatia axilar, distorção arquitetural); F - Forma de localização da lesão (1 - dividir a mama como o mostrador de um relógio, situando a lesão, preferencialmente descrevendo também o quadrante em que ela se situa; 2 profundidade: anterior, média e posterior).

As massas devem ser descritas quanto a: 1 - forma (redonda, ovalada, lobulada e irregular); 2 - margens (circunscrita, microlobulada, obscurecida, indistintas ou mal definidas e espiculadas); 3 - densidade (alta densidade, isodensa e baixa densidade).

As calcificações são enquadradas em 11 grupos: 1 - cutâneas; 2 - vasculares; 3 grosseiras; 4 - em bastonetes; 5 - arredondadas; 6 - com centro radiotransparente; 7 - em casca de ovo; 8 - em leite de cálcio; 9 - calcificações de sutura; 10 - distróficas; 11 - puntiformes. A distribuição descreve o arranjo espacial das calcificações: 1 - agrupadas; 2 - lineares; 3 - segmentares; 4 - regionais; 5 - difusas.

São enquadradas em distorção arquitetural as lesões em que a arquitetura normal da mama está distorcida, mas não há definição de um nódulo. Pode também ser um achado associado.

\section{Seção II - Sistematização do laudo mamográfico}

Esta seção orienta quanto à organização do laudo e quanto à sua redação. Recomenda que o laudo deve ser conciso e organizado, respeitando a seguinte formatação: 1 - fazer referência se o exame sob análise foi comparado a mamografias anteriores; 2 - fazer uma descrição sucinta da composição das mamas, de acordo com quatro padrões preestabelecidos (mamas predominantemente lipossubstituídas, densidades fibroglandulares dispersas, mamas heterogeneamente densas, mamas extrema- mente densas); 3 - fazer descrição clara de qualquer achado significativo (nódulo, calcificações, distorção arquitetural e achados associados); 4 - impressão final. $\mathrm{Na}$ redação do laudo o sistema orienta que os elementos anteriormente citados na organização devem ser referidos no relatório, acompanhados da classificação do exame em uma das seis categorias estabelecidas (categoria 0: necessária avaliação adicional por imagem; categoria 1: negativa; categoria 2: achado benigno; categoria 3: achado provavelmente benigno; categoria 4: anormalidade suspeita; categoria 5: altamente sugestiva de malignidade). Para cada uma destas categorias está inferido um risco de câncer de mama ${ }^{(2,3)}$.

Quando a avaliação é considerada incompleta, o exame é classificado na categoria 0 , recomendando-se avaliação adicional por imagem para subseqüentemente reclassificá-lo em uma das outras cinco categorias. Na avaliação adicional por imagem estão incluídos: comparação com exames anteriores, ultra-sonografia (US), compressão localizada, magnificação e incidências especiais.

Após a interpretação dos achados e a impressão final, devem ser realizadas as recomendações pertinentes. Dessa forma, um laudo poderia ser assim formatado:

- Motivo do exame: rastreamento/ diagnóstico.

- Comparação a exames anteriores.

- Descrição:

a - Constituição das mamas: predominantemente lipossubstituídas/densidades fibroglandulares dispersas/heterogeneamente densas/extremamente densas;

b - Descrição dos achados:

1. MASSAS:

1.1. Forma: redonda/oval/lobulada/irregular.

1.2. Margens: circunscritas/microlobu1adas/obscurecidas/indistintas/espiculadas.

1.3. Densidade: alta densidade/isodensa/hipodensa.

1.4. Localização: usar o marcador de um relógio, precedido por direito, esquerdo, ou ambos, para o lado, preferencialmente citando-se também o quadrante (superior externo ou interno e inferior interno ou externo). Também poderão ser usados subareolar, central ou cauda axilar.
1.5. Profundidade: anterior, médio e posterior.

\section{CALCIFICAÇÕES:}

2.1. Tipo: calcificações de pele/calcificações vasculares/grosseiras/em bastonetes/arredondadas/com centro radiotransparente/em casca de ovo/em leite de cálcio/ calcificações de sutura/calcificações distróficas/puntiformes.

2.2. Distribuição: agrupadas/lineares/ segmentares/regionais/difusas.

\section{DISTORÇÃO ARQUITETURAL}

\section{CASOS ESPECIAIS:}

4.1. Densidade tubular ou ducto solitário dilatado/linfonodo intramamário/tecido mamário assimétrico/densidade assimétrica focal/densidade em uma incidência.

\section{ACHADOS ASSOCIADOS:}

5.1. Retração da pele/retração do mamilo (ou inversão)/espessamento da pele/ espessamento trabecular/lesão da pele/ adenopatia axilar.

\section{- Impressão final:}

Cada lesão descrita deverá ser completamente classificada. Nos casos de exame para rastreamento, o estudo pode ser classificado como inconclusivo se for necessária avaliação adicional por imagem antes que se possa emitir uma impressão final.

Categoria:

- Avaliação incompleta:

Categoria 0: necessária avaliação adicional por imagem (compressão localizada, magnificação, incidências adicionais, US).

- Avaliação completa:

Categoria 1: negativa (não existem alterações suspeitas).

Categoria 2: achado benigno ( achados que merecem ser mencionados, mas são tipicamente benignos - por exemplo, fibroadenomas calcificados, calcificações secretórias, lesões com conteúdo gorduroso, linfonodos intramamários, próteses, etc.).

Categoria 3: achados provavelmente benignos (lesões que não se espera alteração durante o período de observação, mas que o radiologista prefere se certificar de sua estabilidade; possuem alta probabilidade de serem benignas) ${ }^{(\mathbf{1 - 3})}$.

Categoria 4: anormalidade suspeita (estas lesões não possuem características morfológicas compatíveis com câncer, mas têm probabilidade definida de serem malignas) ${ }^{(1-3)}$. 
Categoria 5: altamente sugestiva de malignidade - conduta apropriada deve ser tomada (estas lesões têm alta probabilidade de ser câncer).

\section{- Recomendação:}

Após a impressão final, as recomendações pertinentes devem ser incluídas (por exemplo: categoria 3 - realizar controle em curto intervalo de tempo; categoria 4 biópsia deve ser considerada, etc.).

\section{Objetivos}

Identificar a maneira como vem sendo utilizado o BI-RADSTM , gerando informações que possam auxiliar o Colégio Brasileiro de Radiologia a desenvolver estratégias que possam aperfeiçoar o seu uso no Brasil.

\section{MATERIAIS E MÉTODOS}

Os dados foram coletados em um serviço de radiologia particular na cidade de Goiânia, GO. As mulheres que se dirigiram ao serviço para realização de mamografia, no período de janeiro a junho de 2003 , eram sistematicamente solicitadas a fornecer exames de mamografia anteriores para comparação.

Foram incluídos na análise os exames anteriores realizados entre 1/7/2001 e 30/ 6/2003, quando eram acompanhados dos respectivos laudos. Foram excluídos os exames anteriores que não possuíam seus laudos e aqueles realizados no próprio serviço, para evitar viés.

Os laudos selecionados foram codificados para não haver possibilidade de identificação dos serviços, nem dos médicos responsáveis pela emissão dos laudos. Primeiramente, os laudos eram analisados quanto à adoção do BI-RADS ${ }^{\mathrm{TM}}$. Eram considerados como seguidores do BIRADS $^{\text {TM }}$ os relatórios em que havia tentativa de se enquadrar o resultado em uma das categorias do sistema.

Em seguida os laudos eram avaliados quanto à concisão. Eram considerados concisos os que faziam referência apenas a achados relevantes para a categorização do exame, conforme preconiza o sistema.

O terceiro item avaliado foi a organização. A estrutura do laudo era considerada de acordo com a padronização do BIRADSTM, quando: 1 - fazia referência ao motivo do exame; 2 - fazia referência à comparação com exames prévios; 3 - descrevia a constituição mamária de acordo com as quatro constituições, sem modificar o léxico; 4 - fazia descrição dos achados pertinentes (nódulos, microcalcificações, etc.); 5 - apresentava impressão diagnóstica; 6 - referia a categoria BI-RADS ${ }^{\text {TM} \text {; }}$ 7 - apresentava recomendação de conduta.

Após a análise da estrutura do laudo, avaliava-se a utilização do léxico segundo preconizado no BI-RADS ${ }^{\text {TM }}$. Nos casos em que eram descritos nódulos, era transcrita a denominação dada ao nódulo, a descrição da forma, das margens, do tamanho, da densidade e a localização. Nos casos das microcalcificações, era avaliada a descrição de seus tipos, distribuição e localização.

Em seguida, analisava-se a categoria em que o exame fora classificado (nos que buscavam seguir o BI-RADSTM) e a recomendação de conduta.

Os dados coletados eram arquivados em um banco de dados especialmente desenvolvido no sistema Access 97 da Microsoft $^{\circledR}$.

\section{RESULTADOS}

No total foram fornecidos 104 laudos anteriores, emitidos por 40 radiologistas de 33 diferentes serviços. Destes serviços, 29 eram localizados na própria cidade, dois, em outros estados e dois, em outros países (Chile e Argentina).

Dos 104 laudos, 77\% ( $\mathrm{n}=80)$ utilizavam a categorização BI-RADS ${ }^{\mathrm{TM}}$ no final do relatório. Destes $80,15 \%(n=12)$ eram concisos e nenhum apresentava a estrutura organizacional recomendada no sistema $(\mathrm{n}=0)$.

Daqueles que usavam o BI-RADS ${ }^{\text {TM, }}$ apenas $8,75 \%(n=7)$ faziam referência a exames anteriores para comparação, 2,5\% $(\mathrm{n}=2)$ referiam a indicação clínica, $1,25 \%$ $(\mathrm{n}=1)$ respeitava o léxico e $35 \%(\mathrm{n}=28)$ apresentavam recomendação de conduta.

Os laudos receberam a seguinte categorização BI-RADS ${ }^{\mathrm{TM}}$ : categoria $0-15 \%$ (n $=12)$, sendo que $58,3 \%(\mathrm{n}=7)$ destes receberam esta classificação exclusivamente por se tratarem de mamas densas; categoria $1-37,5 \%(\mathrm{n}=30)$; categoria $2-31,25 \%$ $(\mathrm{n}=25)$; categoria $3-13,75 \%(\mathrm{n}=11)$; categoria $4-2,5 \%(n=2)$. Não houve casos enquadrados na categoria 5.
Nódulos foram descritos em 20\% ( $\mathrm{n}=$ 16) dos laudos que procuravam seguir o BI-RADS ${ }^{\mathrm{TM}}$, os quais receberam as seguintes denominações: nódulo $(n=6 ; 37,5 \%)$, imagem nodular $(\mathrm{n}=4 ; 25 \%)$, imagem radiodensa $(n=1 ; 6,25 \%)$, imagem ovalada $(\mathrm{n}=1 ; 6,25 \%)$, imagem nodulariforme ( $\mathrm{n}$ $=1 ; 6,25 \%)$, assimetria focal nodular $(\mathrm{n}=$ $1 ; 6,25 \%)$ e área nodular $(\mathrm{n}=1 ; 6,25 \%)$. Não havia descrição da forma dos nódulos em $87,5 \%$ dos casos $(n=14)$, das margens em $50 \%(n=8)$ e da densidade em $56,2 \%$ $(\mathrm{n}=9)$. A localização dos nódulos por quadrante foi feita em $87,5 \%$ dos casos (n =14), porém não foi utilizada a localização distribuindo a mama segundo o mostrador de um relógio em nenhum laudo. A descrição da profundidade das lesões também não foi relatada em nenhum dos laudos.

Calcificações foram relatadas em 28,8\% ( $\mathrm{n}=23$ ) dos relatórios que procuravam adotar o BI-RADSTM. O tipo das calcificações não foi mencionado em 8,75\% ( $\mathrm{n}=$ 7), a disposição em $100 \%(n=23)$ e o local em $8,75 \%(n=7)$ dos relatórios.

As recomendações de conduta foram: 1 - para a categoria $0(n=12)$ : US $(n=8)$, compressão localizada ( $\mathrm{n}=1)$, US + punção aspirativa por agulha fina $(\mathrm{n}=1)$, exame que requer complemento $(\mathrm{n}=1)$, não foi citada recomendação $(n=1) ; 2$ - para a categoria $1(\mathrm{n}=30)$ : não foi citada recomendação ( $n=28)$, controle mamográfico anual $(\mathrm{n}=2) ; 3$ - para a categoria 2 $(\mathrm{n}=25)$ : não foi citada recomendação (n =19), controle mamográfico anual $(\mathrm{n}=3)$, repetir de acordo com a faixa etária $(n=1)$, dizia não haver nenhuma recomendação especial $(\mathrm{n}=1)$, mamografia de rotina $+\mathrm{US}$ $(\mathrm{n}=1) ; 4$ - para a categoria $3(\mathrm{n}=11)$ : não havia citação de recomendação $(n=4)$, controle mamográfico dentro de seis meses $(n=3)$, controle mamográfico e exérese $(n=1)$, US $(n=2)$, US + biópsia $(n=$ 1); 5 - para a categoria $4(\mathrm{n}=2)$ : prosseguir investigação $(n=1)$, biópsia $(n=1)$.

A recomendação de conduta não foi citada em $66,6 \%(n=8)$ dos exames classificados na categoria 0 , em 93,3\% $(n=28)$ dos exames classificados na categoria 1 , em $76 \%(n=19)$ dos exames classificados na categoria 2 e em $36,4 \%(n=4)$ dos exames classificados na categoria 3. Ambos os exames classificados na categoria 4 receberam recomendação de conduta. 


\section{DISCUSSÃO}

Aproximadamente $77 \%$ dos laudos analisados se baseavam no BI-RADS ${ }^{\mathrm{TM}}$, indicando sua incorporação no dia-a-dia da interpretação mamográfica. No entanto, a essência do sistema parece não estar clara, já que $85 \%(n=68)$ dos laudos não eram concisos, $100 \%(n=80)$ não se utilizavam da estrutura e organização recomendadas, $87,5 \%(\mathrm{n}=73)$ não faziam referência à comparação com exames anteriores, $97,5 \%$ não mencionavam o motivo do exame e $98,75 \%$ não se utilizavam do léxico. A recomendação de conduta apareceu em apenas $35 \%(n=28)$ dos relatórios. O objetivo central do BI-RADS ${ }^{\mathrm{TM}}$ é promover a padronização do laudo mamográfico, reduzindo os fatores de confusão na interpretação das imagens. Através da padronização dos laudos, são criadas condições para a geração de banco de dados que permitam auditorias médicas e monitoramento eficiente dos resultados.

$\mathrm{O}$ item referente à estrutura e organização dos laudos mamográficos foi o que apresentou maior discrepância entre o preconizado e o realizado. Nenhum dos laudos analisados seguia o padrão (relato de comparação a exames anteriores, quando disponíveis, descrição da composição das mamas, descrição de achados pertinentes e a impressão final com recomendação de conduta). Também foi muito elevado o número de laudos não concisos (85\%).

A baixa submissão ao uso do léxico foi o segundo item mais observado: $98,75 \%$ dos laudos não estavam em conformidade com o seu uso. Dos 16 nódulos descritos por seguidores do BI-RADS ${ }^{\mathrm{TM}}$, apenas seis $(37,5 \%)$ receberam a denominação proposta. Os demais receberam as seguintes denominações: imagem nodular $(25 \% ; \mathrm{n}=$ 4), imagem ovalada $(6,25 \% ; n=1)$, imagem radiodensa $(6,25 \% ; \mathrm{n}=1)$, imagem nodulariforme $(6,25 \% ; \mathrm{n}=1)$, assimetria focal nodular $(6,25 \% ; n=1)$ e área nodu$\operatorname{lar}(6,25 \% ; \mathrm{n}=1)$.

O sistema também regulamenta a descrição das características dos nódulos. Os laudos analisados que adotavam o BIRADS $^{\mathrm{TM}}$ não descreviam a forma dos nódulos em $87,5 \%(\mathrm{n}=14)$ dos casos, as margens em $50 \%(\mathrm{n}=8)$, a densidade em $56,25 \%(\mathrm{n}=9)$ e o tamanho em $50 \%(\mathrm{n}=$
8). A localização dos nódulos foi referida em $87,5 \%(\mathrm{n}=14)$, porém em nenhum deles adotando-se o critério da visualização da mama como o marcador de um relógio. A observação de cada um destes aspectos tem importância na formulação da impressão final, permitindo inferir com maior precisão a classificação final e o respectivo risco de malignidade ${ }^{(1-3)}$.

Calcificações foram relatadas em $28,75 \%(n=23)$ dos laudos nos quais o BIRADS $^{\mathrm{TM}}$ era utilizado, no entanto, o tipo e a localização não foram descritos em $30,4 \%(n=7)$. Na seção do BI-RADS ${ }^{\mathrm{TM}}$ destinada às calcificações, estas são divididas em três tipos: tipicamente benignas, calcificações com grau de suspeição intermediária e com alta probabilidade de malignidade. Esta divisão é baseada na análise conjugada de suas formas, localização e distribuição, justificando a importância da observação de cada um destes itens para a emissão da impressão final.

Recomendação de conduta foi realizada em apenas 35\% dos laudos, constatando-se extrema variação de recomendação para as categorias 0 e 3 (Tabela 1). A categoria 0 foi utilizada para classificar mamas densas em sete casos (representando 58,3\% das categorias 0 ), sendo que, deste total, $57,1 \%(n=4)$ indicavam estudo ecográfico complementar, apesar de não haver descrição de nenhum tipo de alteração. Na categoria 3, apenas $27,3 \%(n=3)$ dos laudos atendiam ao preconizado pelo BI-RADS ${ }^{\mathrm{TM}}$ (exame de controle em curto intervalo de tempo) e, novamente, a US é indicada de forma inadequada: $27,2 \%(n=3)$ dos exames enquadrados nesta categoria receberam recomendação de estudo ecográfico complementar.
A análise global dos dados coletados mostra que nenhum dos laudos que se utilizava do BI-RADS ${ }^{\mathrm{TM}}$ seguia a formatação idealizada pela comissão elaboradora: concisão, organização do laudo (1 - descrição da composição das mamas de acordo com as quatro categorias; 2 - descrição de achados significativos; 3 -impressão final com recomendações de conduta pertinentes) e adoção do léxico. Apesar de a ausência de submissão à estrutura de organização do laudo ser a discordância mais comum, a ausência de adoção do léxico é mais grave, porque compromete qualquer possibilidade de uniformização, já que uma mesma lesão pode ser descrita de formas variadas, como foi verificado na nomenclatura utilizada para a descrição dos nódulos. Ao que tudo indica, perpetua-se o estado de ausência de padronização dos laudos mamográficos, o que motivou a criação do BI-RADS ${ }^{\mathrm{TM}}$.

O estudo tem como limitação o fato de a amostra avaliada ser relativamente pequena, primeiramente devido à dificuldade em se obter os exames anteriores para comparação, e secundariamente porque uma parcela dos exames fornecidos não vinha acompanhada de seus respectivos laudos. Também causou restrição da amostra avaliada a necessidade de se excluir os laudos dos exames anteriores realizados no próprio serviço, para evitar viés. Os resultados, no entanto, são regionalmente representativos, pois, segundo os números da vigilância sanitária local, a cidade possui 36 serviços de mamografia. Dos 33 serviços analisados, 29 eram da própria cidade, portanto, $80,5 \%$ deles participaram da amostra.

Os resultados obtidos corroboram os resultados da avaliação dos laudos mamográficos de 115 serviços de mamografia em

Tabela 1 Ausência de padronização nas recomendações de conduta para as categorias BI-RADS ${ }^{\text {TM }} 0$ e 3.

\begin{tabular}{|l|c|c|}
\hline \multirow{2}{*}{} & \multicolumn{2}{|c|}{ Categoria } \\
\cline { 2 - 3 } & $\mathrm{n}=12(15 \%)$ & $\mathrm{n}=11(13,75 \%)$ \\
\hline Não citava recomendação & $1(8,3 \%)$ & $4(36,4 \%)$ \\
Ultra-sonografia & $8(66,6 \%)$ & $2(18,2 \%)$ \\
Ultra-sonografia + punção aspirativa por agulha fina & $1(8,3 \%)$ & 0 \\
Ultra-sonografia + biópsia & - & $1(9,0 \%)$ \\
Compressão localizada & $1(8,3 \%)$ & 0 \\
Exame que requer complemento (sem especificar qual) & $1(8,3 \%)$ & 0 \\
Controle mamográfico dentro de seis meses & 0 & $3(27,3 \%)$ \\
Controle mamográfico e exérese do nódulo & 0 & $1(9,0 \%)$ \\
\hline
\end{tabular}


todo o Brasil, realizada por Luna e Koch em $1999^{(4,5)}$. Nessa pesquisa, os autores constataram que os laudos estavam sendo realizados com nível científico inadequado e que sua padronização e organização não estavam sendo seguidos pelos serviços.

Estas pesquisas trazem à luz a necessidade de se implementar atividades que proporcionem o reconhecimento do BIRADSTM como um sistema que objetiva muito mais que apenas a classificação final do exame de mamografia.

\section{CONCLUSÕES}

A amostra analisada revela que o BIRADS $^{\text {TM }}$ foi utilizado, quase que exclusi- vamente, como um sistema de classificação final do exame de mamografia. A estrutura, a organização e a forma de redação dos laudos não foram seguidas, na grande maioria dos casos, e o léxico praticamente não foi incorporado aos laudos.

Houve absoluta falta de padronização dos laudos mamográficos em todo o espectro do BI-RADS ${ }^{\mathrm{TM}}$ (estrutura do laudo, comparação a exames anteriores, submissão ao léxico, impressão final e recomendação de conduta), prática que compromete seriamente seus demais propósitos de monitoramento dos resultados, criação de um banco de dados nacional e, por fim, melhoria da qualidade do atendimento prestado às pacientes.

\section{REFERÊNCIAS}

1. American College of Radiology. Breast Imaging Reporting and Data System. BI-RADS ${ }^{\mathrm{TM}}$. 3rd ed. Reston, VA: ACR, 1998.

2. Orel SG, Kay N, Reynolds C, Sullivan DC. BIRADS categorization as a predictor of malignancy. Radiology 1999;211:845-50.

3. Liberman L, Abramson AF, Squires FB, Glassman JR, Morris EA, Dershaw DD. The breast imaging reporting and data system: positive predictive value of mammographic features and final assessment categories. AJR 1998;171:35-40.

4. Luna M, Koch HA. Padronização e organização dos laudos mastográficos, num programa de detecção precoce do câncer de mama. Femina 1999;27:797801.

5. Luna M, Koch HA. Avaliação dos laudos mamográficos: padronização prática de recomendação de conduta para um programa de detecção precoce do câncer de mama por meio da mamografia. Rev Bras Mastol 2002;12:7-12. 